\title{
Nanocompósitos de polietileno/argila bentonítica com propriedades antichama
}

\section{Polyethylene/bentonite clay nanocomposite with flame retardant properties}

\author{
Sara Verusca de Oliveira ${ }^{1}$, Edcleide Maria Araújo ${ }^{1 *}$, Celeste Margarida Correia Pereira ${ }^{2}$ e \\ Amanda Melissa Damião Leite ${ }^{1}$ \\ ${ }^{1}$ Unidade Acadêmica de Engenharia de Materiais, Universidade Federal de Campina Grande - UFCG, \\ Campina Grande, PB, Brasil \\ ${ }^{2}$ Instituto de Engenharia Mecânica e Gestão Industrial - INEGI, Universidade do Porto - UP, Porto, \\ Portugal \\ *edcleide.araujo@ufcg.edu.br
}

\begin{abstract}
Resumo
Nesta pesquisa, nanocompósitos de polietileno de alta densidade (PEAD) com argila montmorilonita (MMT) e argila organofílica (OMMT) foram preparados pelo método de intercalação por fusão, com o objetivo de examinar o efeito antichama do material obtido. Para fins de comparação, utilizou-se um produto comercial retardante de chama (RCP) na matriz de PEAD. O polietileno enxertado com anidrido maleico (PE-g-MA) foi utilizado como um compatibilizante dos sistemas. O PEAD e seus sistemas foram avaliados por difração de raios-X (DRX), inflamabilidade (UL94HB, índice de oxigênio (LOI) e calorimetria de cone). Os difratogramas de raios-X ilustraram que provavelmente ocorreu intercalação e/ou esfoliação parcial, com a formação de nanocompósito e/ou microcompósito. Os resultados obtidos para o índice de oxigênio mostraram que tanto o PEAD como seus sistemas apresentaram propriedades retardantes de chama. Por meio dos ensaios de inflamabilidade horizontal, verificou-se que a presença de $1 \%$ de argila MMT reduziu em $25 \%$ a inflamabilidade de PEAD e, por calorimetria de cone foi visto que a argila OMMT com percentagem de 3 , 6 e $9 \%$ atuou reduzindo também a inflamabilidade dos nanocompósitos.
\end{abstract}

Palavras-chave: nanocompósitos, PEAD, antichama, argila organofilica, calorímetro de cone.

\begin{abstract}
In this research, nanocomposites of high density polyethylene (HDPE) with montmorillonite clay (MMT) and organoclay (OMMT) were prepared by melt intercalation, in order to examine the flame retardant effect of the obtained material. For comparison, a commercial flame retardant product (FRP) was used in the PE matrix. Grafted polyethylene with maleic anhydride (PE-g-MA) was used as a compatibilizer of the systems. HDPE and its systems were evaluated by: X-ray diffraction (XRD), and flammability (UL94HB, oxygen index (LOI) and Cone Calorimetry). The XRD patterns illustrated that probably partial intercalation and exfoliation occurred with the formation of nanocomposite and/or microcomposite. The results obtained for the oxygen index revealed that HDPE and its systems presented flame retardant properties. Through horizontal flammability tests, it was found that the presence of $1 \%$ MMT clay reduced $25 \%$ of the flammability of HDPE. By Cone Calorimetry was found that the OMMT clay with percentage of 3,6 and $9 \%$ acted reducing the flammability of nanocomposites.
\end{abstract}

Keywords: nanocomposite, HDPE, flame retardant, organoclay, cone calorimetry.

\section{Introdução}

Os nanocompósitos poliméricos são uma classe de materiais híbridos compostos de uma matriz polimérica orgânica na qual contém quantidades relativamente pequenas (tipicamente $<10 \%$ ) de nanopartículas inorgânicas dispersas ${ }^{[1-3]}$. Diferentes tipos de cargas em dimensões nanométricas têm sido utilizada para preparar

nanocompósitos sendo: grafite, argila, sílica, nanotubos de carbono entre outras ${ }^{[3,4]}$.

A primeira demonstração prática das vantagens dos nanocompósitos contendo camadas de silicatos dispersas individual e uniformemente em uma matriz polimérica foi feita por pesquisadores da Toyota do Japão usando Nylon- $6^{[5,6]}$. 
Eles obtiveram Nylon-6 através da polimerização intercalativa da $\varepsilon$-caprolactama, resultando em nanocompósitos esfoliados com um aumento significativo do módulo de Young e da tensão de ruptura, mesmo com pouca carga de argila.

Uma das vantagens dos nanocompósitos poliméricos em relação aos compósitos convencionais é que os nanocompósitos podem apresentar propriedades mecânicas e térmicas similares ou superiores aos compósitos convencionais mesmo utilizando uma quantidade baixa de argila. O sucesso na obtenção de tal desempenho consiste na habilidade em esfoliar e dispersar, individualmente, as camadas de silicato, com alto fator de forma, dentro da matriz polimérica ${ }^{[7]}$.

Nanocompósitos poliméricos utilizados para aplicação de materiais retardantes de chama vêm sendo estudados atualmente e um dos maiores obstáculos é o desenvolvimento de nanocompósitos com propriedades antichama capazes de aumentar a resistência físico-química ${ }^{[2]}$.

Dentre os vários tipos de nanocompósitos existentes, os nanocompósitos de polímeros e silicatos lamelares se destacam por suas propriedades mecânicas ${ }^{[8,9]}$, sua resistência ao calor ${ }^{[10]}$ e à oxidação, transparência ótica e biodegradabilidade. Além disso, esses materiais apresentam baixa permeabilidade a gases ${ }^{[8]}$, o que gera perspectiva de aplicação em pneus, embalagens de alimentos e de bebidas gaseificadas e outros artefatos poliméricos nos quais essa propriedade é importante.

A inflamabilidade dos materiais é uma propriedade muito importante. $\mathrm{O}$ entendimento dos processos de combustão, além do comportamento específico do polímero sob interesse, permite o desenvolvimento de alternativas tanto para a redução de sua inflamabilidade quanto da supressão de fumaça produzida durante a combustão. Quando um polímero orgânico é aquecido, ele vai progressivamente sofrendo modificações, em princípio físicas e depois químicas, terminando por sofrer decomposição total em produtos voláteis. Se o polímero tem aditivos minerais como caulim e dióxido de titânio, a combustão total deixa cinzas, nas quais se encontram aqueles aditivos ${ }^{[11]}$.

O processo químico da decomposição de nanocompósitos ocorre quando este é aquecido, dependendo da temperatura e da taxa de aquecimento e da atmosfera. A forma como ocorre o processo pirolítico depende das condições experimentais, das quais as mais importantes são a temperatura de aquecimento $^{[12]}$ e o tipo de polímero usado ${ }^{[13]}$.

O processo de pirólise normalmente ocorre na faixa de temperatura entre $500-800^{\circ} \mathrm{C}$, dependendo do polímero, e em uma rápida taxa de aumento de temperatura. Na maioria dos casos, a pirólise de uma espécie molecular consiste em diversas reações que ocorrem simultaneamente. Depois do primeiro passo da reação pirolítica de um polímero, é comum ocorrerem outras reações subsequentes. Neste caso, o passo inicial da decomposição é seguido por outras reações pirolíticas de moléculas menores, provenientes do composto inicial. Posteriormente os produtos da pirólise provavelmente reagem entre si, gerando novos compostos ${ }^{[12]}$.

Para o PEAD, a pirólise do polietileno em atmosfera inerte inicia em aproximadamente $280^{\circ} \mathrm{C}$ e ocorre principalmente seguindo reações de fragmentação e hidrogenação, a fragmentação se mostra predominante a temperatura abaixo de $600{ }^{\circ} \mathrm{C}$. Os hidrocarbonetos de 2 até 90 carbonos têm sido identificados em pirogramas. Três tipos de moléculas provenientes das reações de fragmentação são as mais comuns, as quais são nomeadas como alcanos, alquenos e $\alpha, \omega$ - dienos. Traços de hidrocarbonetos também podem ser formados durante a pirólise ${ }^{[14]}$.

O mecanismo de decomposição térmica do polietileno começa com uma cisão aleatória, continua com uma reação de propagação que gerará um novo radical polimérico e uma molécula menor e estável e termina com a reação de terminação que também irá gerar uma molécula estável, por exemplo, por desproporcionação com a geração de um alcano e uma $\alpha$-olefina.

A eliminação do etileno (o monômero) não é energicamente favorecida, desta forma ela não ocorrerá. Dienos são formados durante a pirólise com uma $\alpha$-olefina submetendo-se a clivagem, seguida por uma desproporcionação com a formação de um $\alpha, \omega$-dieno. Em altas temperaturas aproximadamente $700-800{ }^{\circ} \mathrm{C}$, traços de hidrocarbonetos aromáticos policíclicos são formados no processo de pirólise. ${ }^{[12]}$

A decomposição de diversos tipos de polietileno com características diferentes, não mostram diferença significativa no processo de decomposição térmica, quando nenhum catalisador é utilizado. No entanto na presença de catalisadores sólidos ácidos, consideráveis diferenças na temperatura de decomposição são evidenciadas ${ }^{[12]}$. E a decomposição do polietileno gera diversos fragmentos moleculares. Para o polietileno de alta densidade (PEAD) aquecido a temperaturas entre $335^{\circ} \mathrm{C}$ e $450{ }^{\circ} \mathrm{C}$, os fragmentos moleculares incluem hidrocarbonetos saturados e hidrocarbonetos mono e di-insaturados provenientes de $\mathrm{C} 2-\mathrm{C} 90$. O aquecimento a temperatura mais baixas mostrou favorecer fragmentos moleculares maiores ${ }^{[12]}$.

Conforme a composição química do polímero, a decomposição térmica pode ser facilitada ou dificultada. Polímeros de fácil decomposição, como o nitrato de celulose, nem permitem a quantificação da propriedade, pela rapidez da combustão. Os polímeros termorrígidos, como as resinas fenólicas, apresentam maior dificuldade de combustão, e por isso são usados na confecção de peças para uso elétrico. Quando o polímero apresenta anéis aromáticos e ausência de cadeias parafínicas há um auto-retardamento da sua inflamabilidade, sem manutenção da chama. A existência de grupos éster favorece o desprendimento de $\mathrm{CO}_{2}$ por aquecimento, contribuindo também para o auto-retardamento da chama ${ }^{[11]}$.

Para nanocompósitos o mecanismo da retardância a chama de polímero/argila é baseado na formação de cinzas. As cinzas isolam a base do polímero do calor formando uma barreira e reduzindo o escape de gases voláteis a partir da combustão do polímero. Embora ainda seja uma área de desenvolvimento relativamente nova, os nanocompósitos de polímero/argila são altamente importantes como um novo sistema de retardantes de chama para polímeros com melhores propriedades. A combinação de argilas organofílicas com outros aditivos retardantes de chama, tais como, a alumina trihidratada, tem demonstrado promissoras propriedades ${ }^{[15]}$.

A resistência ao fogo tem sido melhorada pela utilização de argilas organofílicas em matrizes poliméricas ${ }^{[16]}$. Os nanocompósitos possuem maior estabilidade térmica e menor inflamabilidade quando comparados aos polímeros 
puros e podem apresentar também caráter autoextinguível. A inflamabilidade é melhorada devido à formação de uma camada termicamente isolante pelas nanopartículas da argila organofílica que protege a matriz polimérica e ainda pelo efeito de barreira aos produtos voláteis gerados durante a combustão do nanocompósito impedindo que estes produtos escapem e alimentem a chama. Estes voláteis servem de combustíveis que mantêm a chama durante a combustão ${ }^{[17]}$. A Figura 1 apresenta a função da argila e da estrutura do nanocompósito para a inflamabilidade.

Desta forma, o presente trabalho tem como objetivo principal a avaliação do processo de combustão em nanocompósitos de polietileno de alta densidade possibilitando o desenvolvimento desses materiais com melhores propriedades antichama.

\section{Materiais e Métodos}

\subsection{Materiais}

A argila utilizada para a preparação dos nanocompósitos foi a argila bentonítica sódica, BRASGEL PA, fornecida pela Bentonit União Nordeste, localizada em Campina Grande/PB. A argila foi peneirada para obter granulometria de 200 mesh $(\mathrm{D}=0,074 \mathrm{~mm})$. A capacidade de troca de cátions $(\mathrm{CTC})$ da argila BRASGEL PA é de aproximadamente $90 \mathrm{meq} / 100 \mathrm{~g}$

Para a organofilização da argila foi utilizado o sal quaternário de amônio denominado Praepagen $\mathrm{WB}^{\circledR}$ (cloreto de estearildimetil amônio), fornecido no estado de gel com quantidade de matéria ativa constituída de aproximadamente $75 \%$, contendo cadeias entre 16 e 18 carbonos, fabricado pela Oxiteno.

A matriz empregada neste estudo foi o polietileno de alta densidade de código comercial (JV-060U), fornecida pela BRASKEM (Brasil).

O compatilibizante polar usado é o Polybond 3009, polietileno enxertado com $1 \%$ de anidrido maléico (PE-g-MA), $\mathrm{MFI}=5 \mathrm{~g} / 10 \mathrm{~min}$, fornecido pela Crompton-São Paulo/SP.

O produto comercial retardante de chama utilizado foi o ADK Stabilizer FP-2100J, da ADEKA, à base de nitrogênio-fósforo, que foi nomeado como RCP e fornecido pela BRASKEM (Brasil).

\subsection{Metodologia}

\subsubsection{Preparação da argila organofílica}

Para preparação da argila organofílica com Praepagen $\mathrm{WB}^{\circledR}$, utilizou-se uma dispersão contendo $768 \mathrm{~mL}$ de água destilada e $32 \mathrm{~g}$ de argila. A argila foi adicionada aos poucos com agitação mecânica concomitante e, após a adição de toda a argila, a agitação foi mantida por 20 minutos. Em seguida, foi adicionada uma solução de água destilada e do sal quaternário de amônio. A agitação foi mantida por mais 20 minutos. Feito isso, os recipientes foram fechados e mantidos à temperatura ambiente por 24 horas. Após esse tempo, o material obtido foi filtrado para ser retirado o excesso de sal. A lavagem foi feita com $2.000 \mathrm{~mL}$ de água destilada, empregando Funil de Buchner com kitassato, acoplado a uma bomba de vácuo com pressão de aproximadamente $635 \mathrm{mmHg}$. Os aglomerados obtidos foram secados em estufa a $60^{\circ} \mathrm{C} \pm 5^{\circ} \mathrm{C}$, por um período de 48 horas. Por fim, os aglomerados secos foram desagregados com o auxílio de almofariz até a obtenção de materiais pulverulentos, os quais foram passados em peneira $\mathrm{ABNT} \mathrm{n}^{\circ} 200(\mathrm{D}=0,074 \mathrm{~mm})$ para serem posteriormente caracterizados.

\subsubsection{Preparação dos sistemas PE/argila e PE/aditivo antichama}

Para a obtenção dos sistemas foram preparados inicialmente concentrados de PE-g-MA/argila e PE-g-MA/antichama, em um homogeneizador de alta rotação MH-50. Os concentrados obtidos foram triturados em moinho de facas e, posteriormente, adicionados à matriz polimérica (PEAD), em quantidades necessárias para a obtenção de teores nominais de 1,3,6 e 9\% em peso de argila e/ou antichama e 6\% de PE-g-MA (a quantidade de PE-g-MA foi mantida para os sistemas com argila e com retardante de chama, para efeitos de comparação). Para tanto, foi utilizada uma extrusora de rosca dupla modular, interpenetrante, corrotacional, modelo ZSK 18 da Coperion (Werner \& Pfleiderer), nas seguintes condições: $190{ }^{\circ} \mathrm{C}$ na $1^{\mathrm{a}}$ zona e $210^{\circ} \mathrm{C}$ nas demais zonas, velocidade de $250 \mathrm{rpm}$ e taxa de alimentação $5 \mathrm{Kg} / \mathrm{h}$.

O material extrudado foi granulado e corpos de provas foram moldados por injeção a $210^{\circ} \mathrm{C}$ em uma Injetora, modelo Fluidmec para obtenção de corpos de prova de tração (ASTM D638), impacto (ASTM D256) e inflamabilidade segundo a norma UL94-HB. Corpos de prova com dimensões de aproximadamente $100 \mathrm{~mm}$ x $100 \mathrm{~mm}$ x $5 \mathrm{~mm}$, segundo a norma ASTM E1354-04a, foram produzidos em prensa hidráulica, a $200{ }^{\circ} \mathrm{C}$, para os testes no Calorímetro de Cone.

\subsubsection{Caracterizações}

As análises de difração de raios $\mathrm{X}$ foram conduzidas a temperatura ambiente em um equipamento Shimadzu (XRD-6000) a 40 kV e 30 mA, radiação $\mathrm{Cu} \mathrm{K} \alpha=1,54 \AA$, no intervalo de varredura de $2 \theta$, entre 2 a 30 graus, a uma taxa de $2 \%$ min para os sistemas de nanocompósitos, polietileno puro, argila sem tratamento, argila organofílica e o antichama.

\section{Inicio da chama}
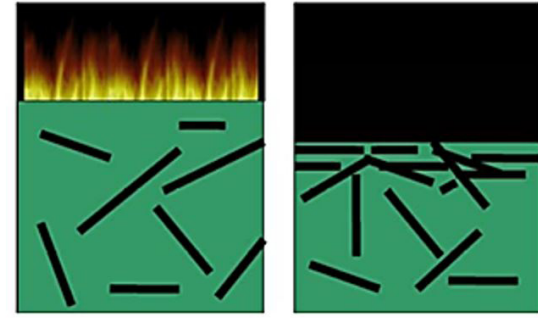

Auto-extinção

Nanocompósito

Figura 1. Função da argila e da estrutura do nanocompósito para a inflamabilidade. Fonte: Barbosa ${ }^{[16]}$ 
Os testes de queima na posição horizontal para determinar a taxa de queima $(\mathrm{mm} / \mathrm{min})$ dos materiais, foram realizados de acordo com a norma UL-94HB.

$\mathrm{O}$ ensaio de índice de oxigênio (LOI) foi realizado de acordo com a ASTM D 2863-95 em um equipamento Fire Testing Technologies em três corpos de prova para cada formulação. Os ensaios foram realizados na temperatura de $(22 \pm 2){ }^{\circ} \mathrm{C}$ e $(53 \pm 2) \%$ de umidade relativa do ar.

$\mathrm{O}$ ensaio no Calorímetro de Cone foi realizado em um equipamento Stanton Redcroft $n^{\circ} 38$ - Fire Testing Technologies de acordo com a norma ASTM E 1354-04a. As propriedades de combustão e emissão de fumaça do PEAD e seus sistemas foram avaliadas em uma média de 3 corpos de prova, para cada composição, sob um fluxo incidente de $50 \mathrm{~kW} / \mathrm{m}^{2}$ e taxa de fluxo no duto igual a $0,025 \mathrm{~m}^{3} / \mathrm{s}$.

\section{Resultados e Discussão}

\subsection{Difração de raios $X(D R X)$}

A Figura 2a-d apresenta os difratogramas de raios $\mathrm{X}$, com $2 \theta$ até $10^{\circ}$, para as argilas, os componentes puros e os sistemas de PEAD com 6\% de PE-g-MA e com 1, 3, 6 e 9\% de argila ou antichama. A ocorrência do processo de intercalação das cadeias poliméricas entre as camadas do silicato pode ser observada através do deslocamento do pico de difração, $\mathrm{d}_{001}$, característico da argila para ângulos menores ( $2 \theta$ apresentado na Figura 2), uma vez que a intercalação conduz a um aumento da distância interplanar basal da argila.

Observando as figuras, pode-se verificar que os nanocompósitos que obtiveram provável intercalação

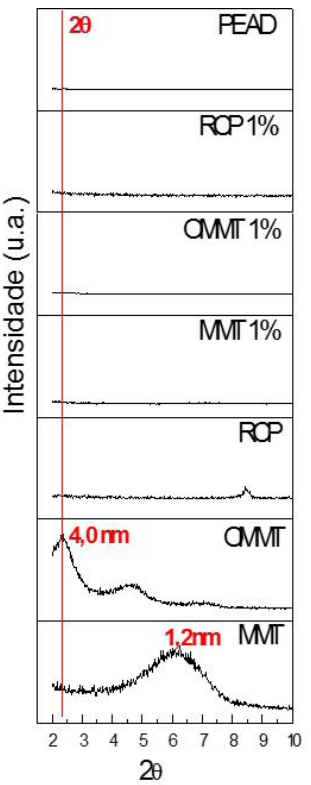

(a)

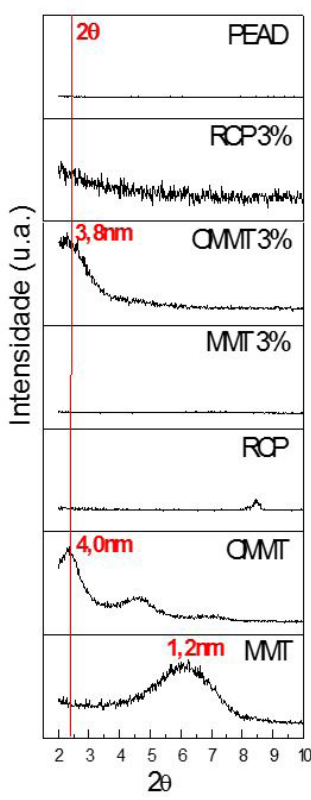

(b) e/ou esfoliação parcial foram aqueles com as composições que continham percentual de 1,3 e 6\% de argila OMMT. Este fato ocorreu devido à modificação química da argila em contato com o sal orgânico. Por meio desses resultados, pode-se evidenciar que a expansão das camadas da argila, influenciou na morfologia dos sistemas e nas propriedades finais dos nanocompósitos. Para o sistema com $9 \%$ de argila, provavelmente ocorreu à formação de microcompósito favorecido por um empilhamento de lamelas, já que possivelmente a interação entre polímero e argila não foi facilitada, conforme também reportado por Cho e Paul ${ }^{[18]} \mathrm{e}$ Barbosa. ${ }^{[16]}$ A não intercalação do polímero entre as lamelas da argila pode ter ocorrido devido ao cisalhamento baixo ocasionado pelo processo de mistura, conduzindo a uma dispersão pouco eficiente da carga na matriz polimérica e/ou pela apolaridade da matriz polimérica. ${ }^{[19]}$

\subsection{Teste de inflamabilidade horizontal UL 94 HB}

A avaliação de inflamabilidade da matriz do PEAD e dos nanocompósitos foi realizada conforme a norma UL-94HB. Neste tipo de teste é muito difícil se obter dados absolutos para todos os materiais em todas as aplicações uma vez que as condições reais onde eles serão exigidos variam bastante $^{[16]}$. A Figura 3 apresenta o comportamento das amostras de nanocompósitos durante o ensaio da UL-94HB.

Observa-se que a maioria dos sistemas apresenta resultados de taxa de queima inferiores ao da matriz polimérica, com exceção do MMT 6\% e OMMT 9\%. Pode-se perceber que as cargas, MMT, OMMT e RCP se comportaram como barreira, provocando um atraso no processo de queima dos sistemas de nanocompósitos. Provavelmente, as cargas atuaram como isolantes aos produtos voláteis de degradação.

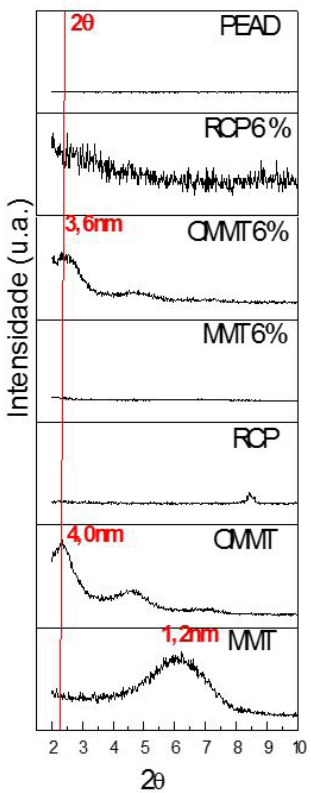

(c)

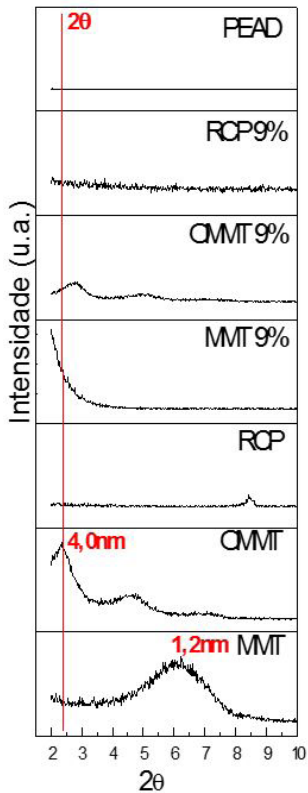

(d)

Figura 2. Difratogramas de raios X das argilas MMT, OMMT, do retardante de chama RCP, do PEAD e seus sistemas com: 1, 3, 6 e $9 \%$ em massa das argilas e do RCP. 


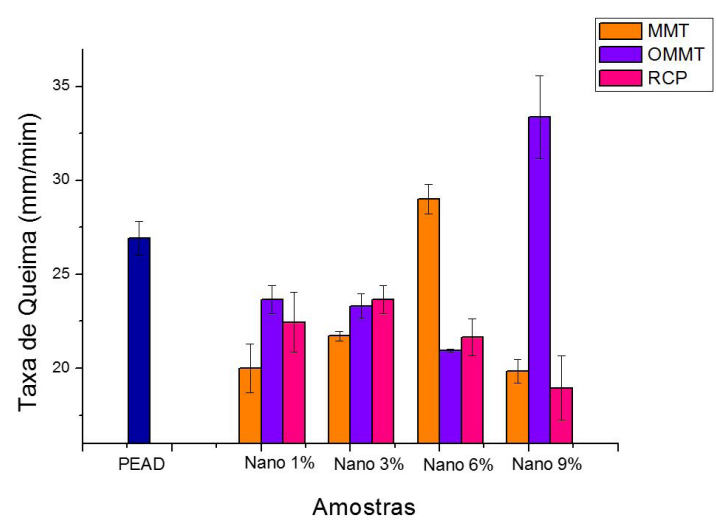

Figura 3. Taxa de queima do PEAD e seus sistemas com: $1,3,6$ e $9 \%$ em massa das argilas e do RCP.

Muito embora, segundo Zhao et al. ${ }^{[20]}$ em estudos com nanocompósitos $\mathrm{PE} /$ argila, foi concluído que a presença da argila pode também provocar a degradação da matriz polimérica, diminuindo a estabilidade térmica.

Verifica-se que os sistemas que apresentam MMT (1,3 e 9\%) em sua composição têm redução de inflamabilidade de aproximadamente 20 a $26 \%$. Para os sistemas com OMMT essa redução fica aproximadamente entre 12 e 22\%. Pelo resultado obtido tem-se que a argila sem modificação química atuou melhor na propriedade antichama o que é bastante significativo para o polietileno de alta densidade, isto é, pode evidenciar que a argila MMT é mais eficaz que a OMMT quanto à propriedade antichama. Observa-se ainda que a diminuição na taxa de queima (aproximadamente 12 e 29\%) para os sistemas com a carga retardante de chama (RCP) é compatível aos sistemas com MMT e que o RCP é mais eficaz com teores maiores. Este resultado é interessante, pois comparando-se o custo de um aditivo retardante de chama (RCP) e que, ao mesmo tempo, é tóxico ao de uma argila regional de baixo custo, natural e de boa qualidade, eleva o valor desta última além de contribuir para a redução do lixo ambiental e da toxicidade deste e proporcionar um material com características antichama.

\section{3 Índice de oxigênio (LOI)}

A Figura 4 ilustra os resultados obtidos no ensaio de LOI apresentados em função do teor das cargas (MMT, OMMT, $\mathrm{RCP})$ na composição dos nanocompósitos e PEAD puro.

Os resultados para o índice de oxigênio mostram que o efeito das cargas (MMT, OMMT e RCP) é pouco significativo quando se busca um elevado índice de oxigênio para retardância à chama, devido os sistemas possuírem valor de LOI inferior a 20,95\%, considerado segundo a norma ASTM D 2863-95 facilmente inflamáveis. Por outro lado, vê-se que quanto maior o teor das cargas, maior a dificuldade de combustão do material. No momento do ensaio foi observado que os sistemas que continham a argila OMMT com carga igual ou superior a $3 \%$ não escorriam nem gotejavam, apenas formavam uma barreira no topo do corpo de prova e a combustão do material era mais lenta.

Pode-se perceber que o PEAD tem valor de LOI aproximadamente $18,3 \%$. Os sistemas que contém cargas

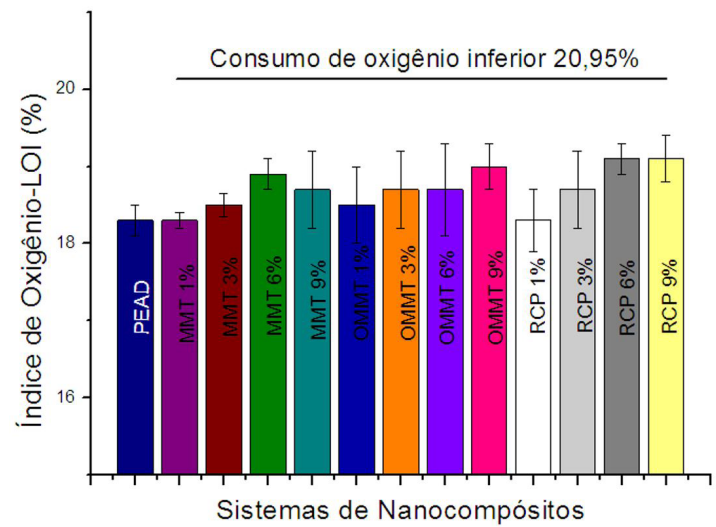

Figura 4. Índice de oxigênio do PEAD e seus sistemas com: $1,3,6$ e $9 \%$ em massa das argilas e do RCP.

MMT, OMMT e RCP têm valor de LOI levemente elevado do que o PEAD puro com exceção do sistema que contém $1 \%$ de MMT. No entanto, o leve aumento do LOI para os sistemas, não foi significativo, segundo a norma ASTM D 2863-95, pois eles consumiram durante a queima um percentual de oxigênio menor do que 20,95\% (Figura 4). A norma referente ao índice de oxigênio (LOI) classifica os nanocompósitos como materiais combustíveis. Por outro lado, os resultados verificados na Figura 3 a partir da UL-94HB são mais significativos, com redução de inflamabilidade de aproximadamente $25 \%$ para o sistema com $1 \%$ de MMT. Por meio da UL-94HB também foi visto que os sistemas que possuem cargas de MMT são menos inflamáveis que os sistemas com OMMT. A adição das cargas conduz um aumento adicional do LOI que relacionado com os resultados da UL94-HB pode-se evidenciar que provavelmente a presença das cargas conduz a uma redução da inflamabilidade do PEAD. Para as nanocargas MMT e OMMT esta redução é devida a acumulação de silicato sobre a superfície da amostra, que forma uma barreira de proteção para a transferência de calor e massa, como reportado por Zanetti e Costa ${ }^{[2]}$ e Peneva et al. ${ }^{[2]}$ Além disso, observou-se também que o PEAD foi queimado a uma taxa mais rápida e com uma chama azul clara. Ao contrário dos sistemas que queimaram mais lentamente e com uma chama brilhante. Os resultados revelam que os nanocompósitos de PEAD apresentam propriedades antichama. Os efeitos são mais pronunciados para nanocompósitos que contém cargas MMT.

Lenza et al. ${ }^{[23]}$ estudaram sistemas de PEAD com $1,2,3,4$ e $5 \%$ de MMT e OMMT e um retardante de chama a base de hidróxido de magnésio com altos teores (em torno de 50\%). Os resultados do PEAD e dos nanocompósitos com MMT e OMMT foram semelhantes aos obtidos nesta pesquisa, com valores de LOI em torno de $20 \%$. Eles verificaram ainda que a adição da argila OMMT favoreceu a valores de LOI um pouco maiores que os obtidos para os sistemas com MMT, como visto também neste trabalho. Entretanto, os maiores valores de LOI, em torno de $29 \%$, foram obtidos para os sistemas com altos teores de hidróxido de magnésio, ou seja, pequenos teores das cargas de MMT e OMMT (1 a 5\%), não apresentaram resultados significativos de LOI. Eles concluíram que altos teores de retardante de chama são necessários para se conseguir um material auto-extinguível. 


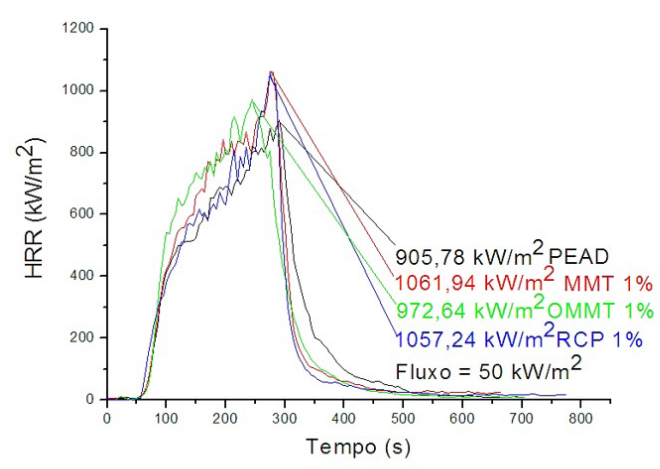

(a)

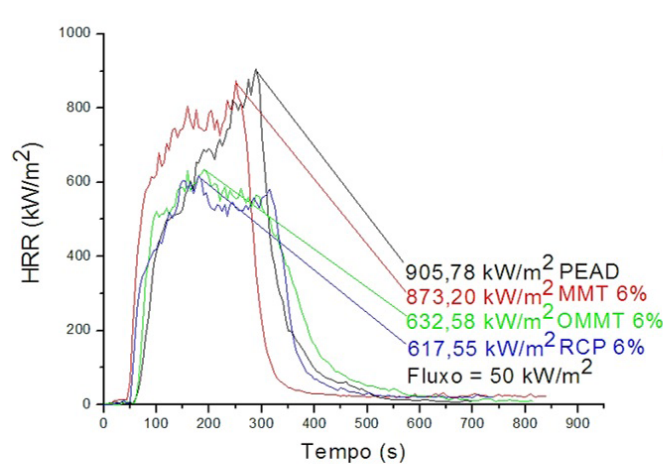

(c)

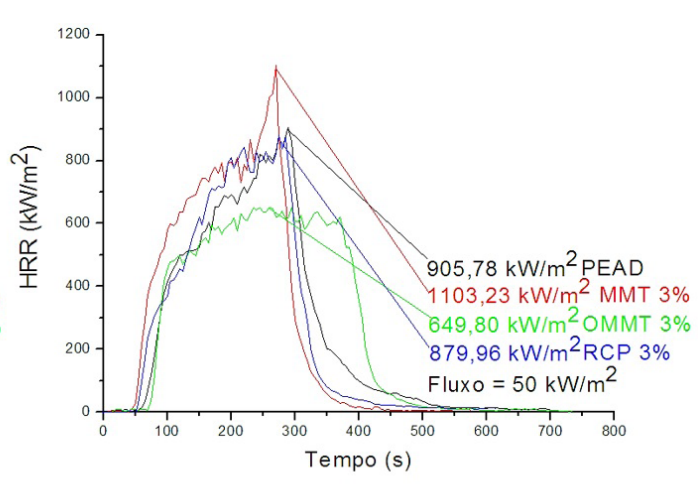

(b)

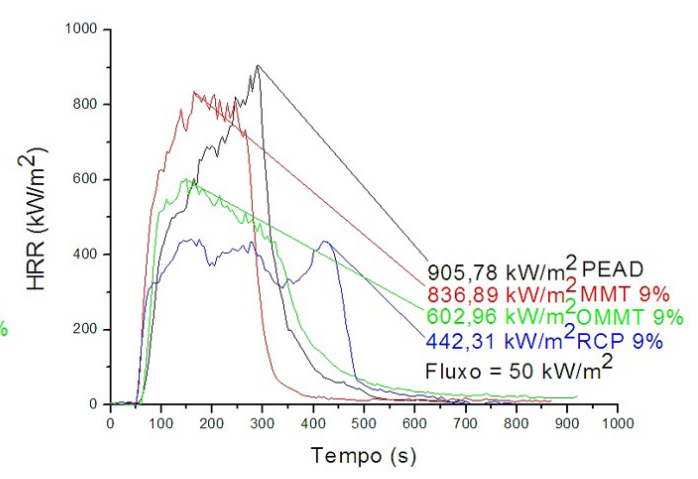

(d)

Figura 5. Curvas de taxa de liberação de calor (HRR) do PEAD e seus sistemas com: (a) 1, (b) 3, (c) 6 e (d) $9 \%$ em massa das argilas e do RCP.

\subsection{Calorímetro de cone}

O calorímetro de cone é uma das mais eficazes caracterizações de inflamabilidade, em caso de incêndios e similares. Os ensaios foram realizados segundo os critérios da norma ASTM E 1354-04. Foi medida a taxa de liberação de calor (HRR), para todos os nanocompósitos com fluxo de calor incidente de $50 \mathrm{~kW} / \mathrm{m}^{2}$. A Figura 5 apresenta as taxas de liberação de calor (HRR), em função do tempo, obtidas a partir do calorímetro de cone para o polietileno de alta densidade e seus sistemas.

Verifica-se que todos os sistemas com percentual de $1 \%$ de carga (Figura 5a) apresentaram um aumento de taxa de liberação de calor em relação à matriz polimérica, reportando assim que estes sistemas não apresentam propriedade antichama. Para os sistemas com 3\% de carga, observa-se que a OMMT apresenta menor taxa de liberação de calor, consequentemente, pode promover uma diminuição na combustão em relação ao PEAD puro. Para 6\% e 9\% de carga, todos os sistemas têm inflamabilidade inferior ao polímero puro como se pode observar na Figura $5 \mathrm{c}-\mathrm{d}$ e, as cargas que atuam diminuindo a inflamabilidade dos sistemas são a OMMT e RCP. O fato da carga OMMT contribuir com a propriedade antichama, foi observado também por estudos realizados por Song et al. ${ }^{[24]}$ e Barbosa ${ }^{[16]}$ que estudaram propriedades de inflamabilidade em nanocompósitos de PE com argilas sem modificação e organofílicas e chegaram a conclusão que a incorporação de argilas organofílicas cria uma camada protetora carbonácea. Comparando a taxa de liberação de calor de todos os sistemas com o PEAD, observa-se que aqueles com 3, 6 e 9\% de OMMT e RCP têm propriedade de inflamabilidade melhor que os demais. Pode-se argumentar que segundo os resultados obtidos por calorimetria de cone, faz-se necessário organofilizar a argila que será incorporada ao polímero para reduzir a inflamabilidade de nanocompósitos de PEAD. A redução do pico HRR dos sistemas com cargas OMMT e RCP é bastante significativa em relação ao PEAD. A Tabela 1 apresenta os parâmetros: o tempo de ignição $\left(\mathrm{t}_{\mathrm{ign}}\right)$, a taxa de liberação de calor (HRR) e o total de calor liberado (THR) do PEAD e seus sistemas.

O tempo de ignição ( $\mathrm{t}_{\mathrm{ign}}$ ) o tempo de exposição mínimo necessário para a amostra acender e manter a chama até a combustão. Redução significativa no pico HRR e o mais longo tempo de ignição foram observados para o PEAD com 3\% OMMT que indica uma melhoria no retardamento da chama. O valor de pico HRR $\left(649,8 \mathrm{~kW} / \mathrm{m}^{2}\right)$ da amostra apresentada tem cerca de $28,2 \%$ de redução em relação ao polietileno de alta densidade ${ }^{[23]}$. Este fato é interessante porque se utilizou um baixo teor de uma carga de abundância regional, não tóxica e de baixo custo, as chamadas argilas bentoníticas. 
Tabela 1. Dados obtidos no ensaio de Calorimetria de Cone em fluxo de calor incidente de $50 \mathrm{~kW} / \mathrm{m}^{2}$, para o PEAD e seus sistemas.

\begin{tabular}{|c|c|c|c|c|c|c|}
\hline Amostras & $\begin{array}{l}t_{\text {ign }} \\
(s)\end{array}$ & $\begin{array}{c}\text { HRR } \\
\left(\mathrm{kW} / \mathrm{m}^{2}\right)\end{array}$ & $\begin{array}{l}\mathbf{t}_{\mathrm{HRR}} \\
(\mathrm{s})\end{array}$ & $\begin{array}{l}\text { Redução } \\
\text { HRR (\%) }\end{array}$ & $\begin{array}{c}\text { Média HRR } \\
\left(\mathbf{k W} / \mathbf{m}^{2}\right)\end{array}$ & $\begin{array}{c}\text { Total THR } \\
\left(\mathrm{MJ} / \mathbf{m}^{2}\right)\end{array}$ \\
\hline PEAD & 57 & 905,8 & 290 & - & 349,9 & 176,8 \\
\hline MMT 1\% & 52 & 1061,9 & 275 & - & 296,9 & 181,2 \\
\hline MMT 3\% & 42 & 1103,2 & 270 & - & 336,7 & 178,5 \\
\hline MMT 6\% & 40 & 873,2 & 250 & 3,6 & 228,3 & 182,6 \\
\hline MMT 9\% & 49 & 836,9 & 165 & 7,5 & 212,6 & 174,3 \\
\hline OMMT 1\% & 61 & 972,6 & 245 & - & 280,2 & 179,4 \\
\hline OMMT 3\% & 67 & 649,8 & 245 & 28,2 & 286,5 & 190,5 \\
\hline OMMT $6 \%$ & 50 & 632,6 & 190 & 30,1 & 231,7 & 177,2 \\
\hline OMMT 9\% & 51 & 602,9 & 150 & 33,2 & 198,7 & 171,9 \\
\hline RCP $1 \%$ & 52 & 1057,2 & 275 & - & 231,1 & 166,4 \\
\hline RCP $3 \%$ & 46 & 879,9 & 275 & 2,7 & 299,8 & 169,4 \\
\hline RCP 6\% & 42 & 617,6 & 180 & 31,7 & 235,0 & 163,4 \\
\hline RCP 9\% & 46 & 442,3 & 175 & 51,2 & 218,6 & 161,8 \\
\hline
\end{tabular}

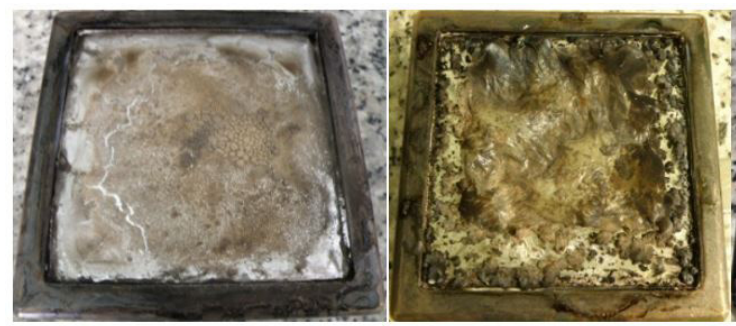

(a) $1 \% \mathrm{MMT}$ (b) $1 \%$ OMMT

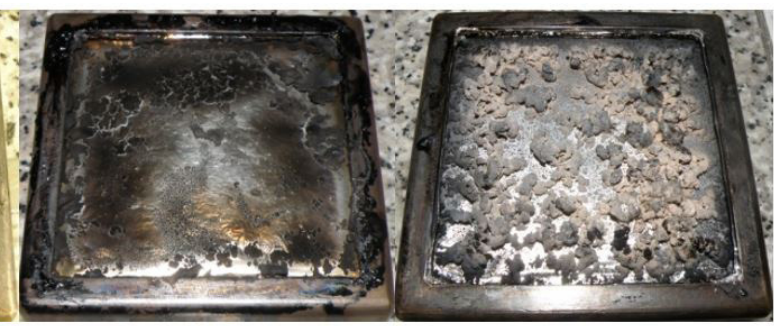

(c) $1 \% \mathrm{RCP}$

(d) $3 \%$ OMMT

Figura 6. Macroimagens dos resíduos dos sistemas de PEAD: (a) 1\% MMT, (b) 1\% OMMT, (c) 1\% RCP e (d) 3\% OMMT.

Com o aumento do teor de carga, tanto a OMMT quanto o RCP, os sistemas mostraram uma diminuição do valor de um parâmetro importante para a segurança contra incêndio que é o pico HRR. Além do HRR, outro parâmetro de incêndio considerado importante, é o menor valor do total de calor liberado (THR) que foi obtido utilizando 9\% RCP. Também nos testes de LOI e UL-94-HB, observa-se que o aumento do teor das cargas reduz a inflamabilidade dos nanocompósitos. Como já mencionado anteriormente, a literatura reporta que altos teores de retardante de chama são necessários para se conseguir um material auto-extinguível ${ }^{[23,25]}$. A Figura 6 ilustra as macroimagens dos resíduos carbonáceos de nanocompósitos de PEAD com 1\%MMT, 1\%OMMT, $1 \%$ RCP e $3 \% \mathrm{OMMT}$ que permaneceram após o ensaio no Calorímetro de Cone.

Para explicar como a formação do resíduo carbonáceo afeta a combustão dos sistemas de PEAD com propriedades antichama, foram registrados por meio de fotos em uma câmera digital, os resíduos obtidos após o ensaio no Calorímetro de Cone. A diferença aparece entre o resíduo do produto comercial (RCP) e da argila (MMT e OMMT), conforme ilustrados na Figura 6. Para o RCP quase não se observa esta camada, sendo que para as amostras com OMMT esta camada é bem expressiva. Este fato também foi observado por Lenza et al. ${ }^{[23]}$ que estudaram sistemas de PEAD com argila. A partir da estrutura carbonácea se pode explicar o fenômeno de combustão dos nanocompósitos de
PEAD com OMMT. A formação do resíduo carbonáceo pode evitar a transferência de calor entre a zona da chama e o material a ser queimado e assim proteger essa parte que ainda não entrou em combustão da outra que ainda está em chamas, atuando como uma barreira e retardando a pirólise do polímero. Como resultado, os valores de picos HRR dos nanocompósitos que contém OMMT são altamente reduzidos quando comparados ao polímero puro (Tabela 1).

\section{Conclusões}

Nanocompósitos de PEAD com argila montmorilonita (MMT) e argila organofílica (OMMT) foram obtidos por intercalação por fusão. A fim de comparar a retardância à chama destes materiais, um produto comercial retardante de chama (RCP) foi utilizado na matriz de PEAD. Por DRX foi verificada a eficiência no processo de organofilização da argila, sendo este verificado pela expansão basal da argila OMMT. Para os nanocompósitos, foi observado por DRX, uma provável intercalação e/ou esfoliação parcial para os sistemas com 1, 3 e 6\% de argila e a formação de microcompósito para o teor de $9 \%$ de argila. Os resultados obtidos para o índice de oxigênio (LOI) ilustraram que os nanocompósitos de PEAD apresentaram propriedades antichama e que a presença de 1\% de argila MMT reduziu em 25\% a inflamabilidade do PEAD, nos testes de inflamabilidade horizontal. Os resultados do Calorímetro 
de Cone ilustraram que a argila OMMT atuou reduzindo a inflamabilidade do PEAD, que pôde ser visto também por meio das macroimagens que apresentaram uma maior quantidade de resíduos carbonáceos.

\section{Agradecimentos}

Os autores agradecem à Braskem, Oxiteno, Bentonit União Nordeste e Crompton por ter disponibilizado todo o material utilizado na pesquisa. Ao DEMa/UFCG, DEMa/UFSCar e ao INEGI (Instituto de interface da Universidade do Porto/Portugal) pelo uso de seus laboratórios. Ao PDSE/CAPES e ao MCTI/CNPq pelo apoio financeiro.

\section{Referências}

1. Giannelis, E. P. (1996). Polymer layered nanocomposites. Advanced Materials, 8(1), 29-35. http://dx.doi.org/10.1002/ adma.19960080104.

2. Zhu, J., Uhl, F. M., Morgan, A. B., \& Wilkie, C. A. (2001). Studies on the mechanism by which the formation of nanocomposites enhances thermal stability. Chemistry of Materials, 13(12), 4649-4654. http://dx.doi.org/10.1021/cm010451y.

3. Linhares, A., Canalda, J. C., Cagiao, M. E., Gutierrez, M. C. G., Nogales, A., Gullón, I. M., Vera, J., \& Ezquerra, T. A. (2007). Broad-band electrical conductivity of high density polyethylene nanocomposites with carbon nanoadditives: multiwall carbon nanotubes and carbon nanofibers. Macromolecules, 40(19), 8501-8517. http://dx.doi.org/10.1021/ma801410j.

4. Gopakumar, T. G., \& Pagé, D. J. Y. S. (2004). Polyprolylene/ graphite nanocomposites by thermo-kinetic mixing. Polymer Engineering and Science, 44(6), 1162-1169. http://dx.doi. org/10.1002/pen.20109.

5. Okada, A., Fukushima, Y., Kawasumi, M., Inagaki, S., Usuki, A., Sugiyama, S., Kurauchi, T., \& Kamigaito, O. (1988). US Patent No 4.739.007.

6. Usuki, A., Kawasumi, M., Kojima, Y., Okada, A., Kurauchi, T., \& Kamigaito, O. (1993). Swelling behavior of montmorillonite cation exchanged for $\omega$-amino acids by $\varepsilon$ - caprolactam. Journal of Materials Research, 8(5), 1174-1178. http://dx.doi. org/10.1557/JMR.1993.1174.

7. Wang, Y., Chen, F. B., Li, Y. C., \& Wu, K. C. (2004). Melt processing of polypropylene/clay modified with maleated polypropylene compatibilizer. Composites. Part B, Engineering, 35(2), 111-124. http://dx.doi.org/10.1016/S1359-8368(03)00049-0.

8. Kojima, Y., Usuki, A., Kawasumi, M., Okada, A., Kurauchi, T., \& Kamigaito, O. (1993). One-pot synthesis of nylon 6-clay hybrid. Journal Polymer Science, 31(7), 1755-1758. http:// dx.doi.org/10.1002/pola.1993.080310714.

9. Varghese, S., Gatos, K. G., Apostolov, A. A., \& Karger-Kocsis, J. (2004). Morphology and mechanical properties of layered silicate reinforced natural and polyurethane rubber blends produced by latex compounding. Journal of Applied Polymer Science, 92(1), 543-551. http://dx.doi.org/10.1002/app.20036.

10. Gilman, J. W., Jackson, C. L., Morgan, A. B., Harris, R. J., Manias, E., Giannelis, E. P., Wuthenow, M., Hilton, D., \& Phillips, S. H. (2000). Flammability properties of polymerlayered-silicate nanocomposites. Polypropylene and polystyrene nanocomposites. Chemistry of Materials, 12(7), 1866-1873. http://dx.doi.org/10.1021/cm0001760.

11. Canaud, C., Visconte, L. L. Y., \& Nunes, R. C. R. (2004). Propriedades mecânicas e de inflamabilidade de composições de borracha EPDM carregadas com negro de fumo e hidróxido de alumínio. Polímeros: Ciência e Tecnologia, 11(1), 35-40. http://dx.doi.org/10.1590/S0104-14282001000100009.

12. Moldoveanu, S. C. (2005). Analytical pyrolysis of synthetic organic polymers. Amsterdam: Elsevier.

13. Pierella, L. B., Renzini, S., \& Anunziata, O. A. (2005). Catalytic degradation of high density polyethylene over microporous and mesoporous materials. Microporous and Mesoporous Materials, 81(1-3), 155-159. http://dx.doi.org/10.1016/j. micromeso.2004.11.015.

14. Kahlow, S. (2007). Pirólise de polipropileno pós-consumo visando a obtenção de novos produtos (Dissertação de mestrado). Universidade Tecnológica Federal do Paraná, Curitiba.

15. European Brominated Flame Retardant Industry Panel-EBFRIP. (2000). Bromine Science and Environmental Forum - BSEF. Brussels, Belgium: EBFRIP. Recuperado em 10 de julho de 2010, de http://www.ebfrip.org

16. Barbosa, R. (2009) Estudo da modificação de argilas bentoníticas para aplicação em nanocompósito de polietileno (Tese de doutorado). Universidade Federal de Campina Grande, Campina Grande.

17. Ray, S. S., \& Okamoto, M. (2003). Polymer/layered silicate nanocomposites: a review from preparation to processing. Progress in Polymer Science, 28(11), 1539-1641. http://dx.doi. org/10.1016/j.progpolymsci.2003.08.002.

18. Cho, J. W., \& Paul, D. R. (2001). Nylon 6 nanocomposites by melt compounding. Polymer, 42(3), 1083-1094. http://dx.doi. org/10.1016/S0032-3861(00)00380-3.

19. Kissin, Y. V., \& Fruitwala, H. A. (2007). Analysis of polyolefins and olefin copolymers using Crystal technique: resolution of Crystal curves. Journal of Applied Polymer Science, 106(6), 3872-3883. http://dx.doi.org/10.1002/app.27090.

20. Zhao, Z., Tang, T., Qin, Y., \& Huang, B. (2003). Effects of surfactant loadings on the dispersion of clays in maleated polypropylene. Langmuir, 19(18), 7157-7159. http://dx.doi. org/10.1021/la034575w.

21. Zanetti, M., \& Costa, L. (2004). Preparation and combustion behaviour of polymer/layered silicate nanocomposites based upon PE and EVA. Polymer, 45(13), 4367-4373. http://dx.doi. org/10.1016/j.polymer.2004.04.043.

22. Peneva, Y., Tashev, E., \& Minkova, L. (2006). Flammability, micro hardness and transparency of nanocomposites based on functionalized polyethylenes. European Polymer Journal, 42(10), 2228-2235. http://dx.doi.org/10.1016/j.eurpolymj.2006.06.027.

23. Lenza, J., Merkel, K., \& Rydarowski, H. (2012). Comparison of the effect of montmorillonite, magnesium hydroxide and a mixture of both on the flammability properties and mechanism of charformation of HDPE composites. Polymer Degradation \& Stability, 97(12), 2581-2593. http://dx.doi.org/10.1016/j. polymdegradstab.2012.07.010.

24. Song, R., Wang, Z., Meng, B., Zhang, T., \& Tang, T. (2007). Influences of catalysis and dispersion of organically modified montmorillonite on flame retardancy of polypropylene nanocomposites. Journal of Applied Polymer Science, 106(5), 3488-3494. http://dx.doi.org/10.1002/app.27033.

25. Alves, T. S., Barbosa, R., Carvalho, L. H., \& Canedo, E. L. (2014). Inflamabilidade de nanocompósitos de polipropileno/ argila organofílica. Polímeros: Ciência e Tecnologia, 24(3), 307-313. http://dx.doi.org/10.4322/polimeros.2014.030.

Enviado: Set. 03, 2015

Revisado: Abr. 22, 2016

Aceito: Abr. 28, 2016 